\title{
ReseqChip: Automated integration of multiple local context probe data from the MitoChip array in mitochondrial DNA sequence
} assembly

\author{
Marian Thieme*1, Claudio Lottaz ${ }^{1}$, Harald Niederstätter ${ }^{2}$, Walther Parson ${ }^{2}$, \\ Rainer Spang ${ }^{1}$ and Peter J Oefner ${ }^{1}$
}

Address: ${ }^{1}$ Institute of Functional Genomics, University of Regensburg, Josef-Engert-Str. 9, D-93053 Regensburg, Germany and ${ }^{2}$ Institute of Legal Medicine, Innsbruck Medical University, Muellerstrasse 44, A-6020 Innsbruck, Austria

Email: Marian Thieme* - marian.thieme@klinik.uni-regensburg.de; Claudio Lottaz - claudio.lottaz@klinik.uni-regensburg.de;

Harald Niederstätter - harald.niederstaetter@i-med.ac.at; Walther Parson - walther.parson@i-med.ac.at; Rainer Spang - rainer.spang@klinik.unir.de; Peter J Oefner - peter.oefner@klinik.uni-regensburg.de

* Corresponding author

Published: 22 December 2009

BMC Bioinformatics 2009, 10:440 doi:10.1/86/147|-2105-10-440

This article is available from: http://www.biomedcentral.com//47I-2/05/10/440

(c) 2009 Thieme et al; licensee BioMed Central Ltd.

This is an Open Access article distributed under the terms of the Creative Commons Attribution License (http://creativecommons.org/licenses/by/2.0), which permits unrestricted use, distribution, and reproduction in any medium, provided the original work is properly cited.
Received: 26 June 2009

Accepted: 22 December 2009

\begin{abstract}
Background: The Affymetrix MitoChip v2.0 is an oligonucleotide tiling array for the resequencing of the human mitochondrial $(\mathrm{mt})$ genome. For each of 16,569 nucleotide positions of the $\mathrm{mt}$ genome it holds two sets of four 25-mer probes each that match the heavy and the light strand of a reference $\mathrm{mt}$ genome and vary only at their central position to interrogate all four possible alleles. In addition, the MitoChip v2.0 carries alternative local context probes to account for known $m t D N A$ variants. These probes have been neglected in most studies due to the lack of software for their automated analysis.

Results: We provide ReseqChip, a free software that automates the process of resequencing mtDNA using multiple local context probes on the MitoChip v2.0. ReseqChip significantly improves base call rate and sequence accuracy. ReseqChip is available at http://code.open-bio.org/ svnweb/index.cgi/bioperl/browse/bioperl-live/trunk/Bio/Microarray/Tools/.
\end{abstract}

Conclusions: ReseqChip allows for the automated consolidation of base calls from alternative local $\mathrm{mt}$ genome context probes. It thereby improves the accuracy of resequencing, while reducing the number of non-called bases.

\section{Background}

The human mitochondrial (mt) DNA is a doublestranded circular molecule of 16,569 base pairs (bp) and consists of two parts: The non-coding displacement loop, also referred to as the control region, and the coding region. The control region is $1,124 \mathrm{bp}$ in size and encompasses the nucleotide positions (nps) 16,024 to 576 . It contains transcription and replication elements. The hypervariable segments HVS I (nps 16,024-16,383) and HVS II (nps 57-372) within the control region are hotspots for mtDNA alterations. The mutation rate of the hypervariable segments is tenfold higher than that of the coding region [1], whose mutation rate is already 10 times higher than that of nuclear genomic DNA because of the lack of protective histones, inefficient DNA repair systems and continuous exposure to mutagenic effects of oxygen 
radicals generated by oxidative phosphorylation [2]. The mtDNA coding region, on the other hand, contains 37 genes coding for two ribosomal RNAs and 22 transfer RNAs, which are required for intramitochondrial translation, as well as 13 polypeptides, which are components of the respiratory chain enzyme complexes in the inner membrane of the mitochondria that are essential in the energy production of the human cell. While most human cells contain two copies of nuclear DNA, they may possess up to 100,000 copies of mtDNA [3]. The majority of these copies are identical or homoplasmic immediately after birth. Somatic mutations will give rise to variant mtDNA copies or heteroplasmy over a lifetime, possibly contributing, for example, to the initiation and progression of cancer [4]. Mutant copies may also be inherited and are known to cause a variety of diseases affecting mostly the energy-hungry cells of the muscles, brain, or nerves [5]. The large number of copies and polymorphisms has made mtDNA particularly useful in forensics [6] and paleogenetics [7] where the amount of nuclear DNA in a sample is limited or degraded. Finally, due to its maternal inheritance and the lack of recombination, mtDNA provides insights into the maternal history of anatomically modern humans and into the impact of genetic drift, demography and selection on the level, kind and distribution of polymorphism observed in extant mtDNA lineages [8]. The analysis of mtDNA sequence variation is therefore of common interest in medical, forensic and population genetics. Re-sequencing of mtDNA has been traditionally accomplished by PCR amplification of the mitochondrial genome in approximately 40 overlapping fragments followed by uni- or preferentially bidirectional sequencing [8]. Although modern capillary array sequencers allow the analysis of as many as 2,300 sequencing reactions or an equivalent of 29 mitochondrial genomes per day, the complexity of conventional sequence analysis has spurred the development of novel tools such as the Affymetrix oligonucleotide-based array MitoChip for the detection of $m t D N A$ variation. The first version of MitoChip was introduced in 2004. It was capable of sequencing the mtDNA coding region in a single hybridization after its long-range amplification in three overlapping fragments [9]. A more recent version (v2.0) of MitoChip covers in addition the control region. More importantly, it carries probes that match not only a reference mtDNA (the so-called revised Cambridge Reference Sequence (rCRS) [10]), but also common variants thereof, $99 \%$ of which are located in the hypervariable segments of the control region. The MitoChip v2.0 has been applied mainly to the detection of somatic mutations in cancer [11-17]. In addition, it has been used in forensic and population genetic studies $[18,19]$.

As illustrated in Figure 1A, microarray-based re-sequencing is done in a local sequence context. Every base of the $\mathrm{mt}$ genome is interrogated by hybridization of fluorescent-labeled DNA fragments against 8 different 25-mer probes forming two probe quartets, one quartet for the light (forward) and one for the heavy (reverse) mtDNA strand. These probes vary only at their central nucleotide position (grey bars with letter in the middle) to account for the 4 possible alleles A, T, C, G or combinations thereof. The 12 bases up-and downstream of the variable position match the rCRS [10]. They constitute the local sequence background for hybridization. Polymorphisms within the flanking nps compromise hybridization. Due to the high mutation rate, multiple polymorphic sites within a window of $25 \mathrm{nps}$ are more frequent in the hypervariable than the control and coding regions. To address the problem of compromised hybridization, the MitoChip v2.0 carries additional alternative probes that match not only the rCRS, but also common variants thereof. The MitoChip v2.0 systematically probes for known hybridization context alternatives including insertions, deletions, and closely spaced single nucleotide polymorphisms. These additional probes embed their middle position not into the context of the reference sequence, but into the context of known variants using alternative flanking probe sequences. Figure 2 illustrates the number of different contexts, available for each position that is covered by at least one additional context.

The alternative context probes, however, have been mostly ignored to date in studies employing the MitoChip v2.0 $[11-18,20,21]$. This has been due to the lack of software for the automated analysis of multiple local context probe data.

Resequencing analysis of $\mathrm{mt}$ genomes operates in two modes: In the qualitative mode, a genotype, namely $\mathrm{A}, \mathrm{C}$, G, T, AC, AG, AT, CG, CT, GT, ACG, ACT, AGT, CGT, ACGT or N, is called for each nucleotide position (np). In the quantitative mode, allele proportions are determined in case of heteroplasmic nps. The challenge in both modes is to improve the analysis by using alternative local context probe information.

For nuclear DNA, Two software tools, namely the RATools and the Affymetrix GeneChip Sequence Analysis Software (GSEQ), are used to call bases from hybridization signal intensities. Both are based on the ABACUS algorithm [22]. Position by position, ABACUS screens a sequence of fluorescence intensities and calculates likelihoods for each base independently for both the forward and the reverse strand. For haploid data, five possible calls (Null, A, C, G, T) are examined. For diploid data 6 possible diploid calls (AC, AG, AT, CG, CT, GT) are considered in addition. The pixel intensities within a probe are modeled as independent random variables with a common mean and variance. Means and variances are estimated by max- 


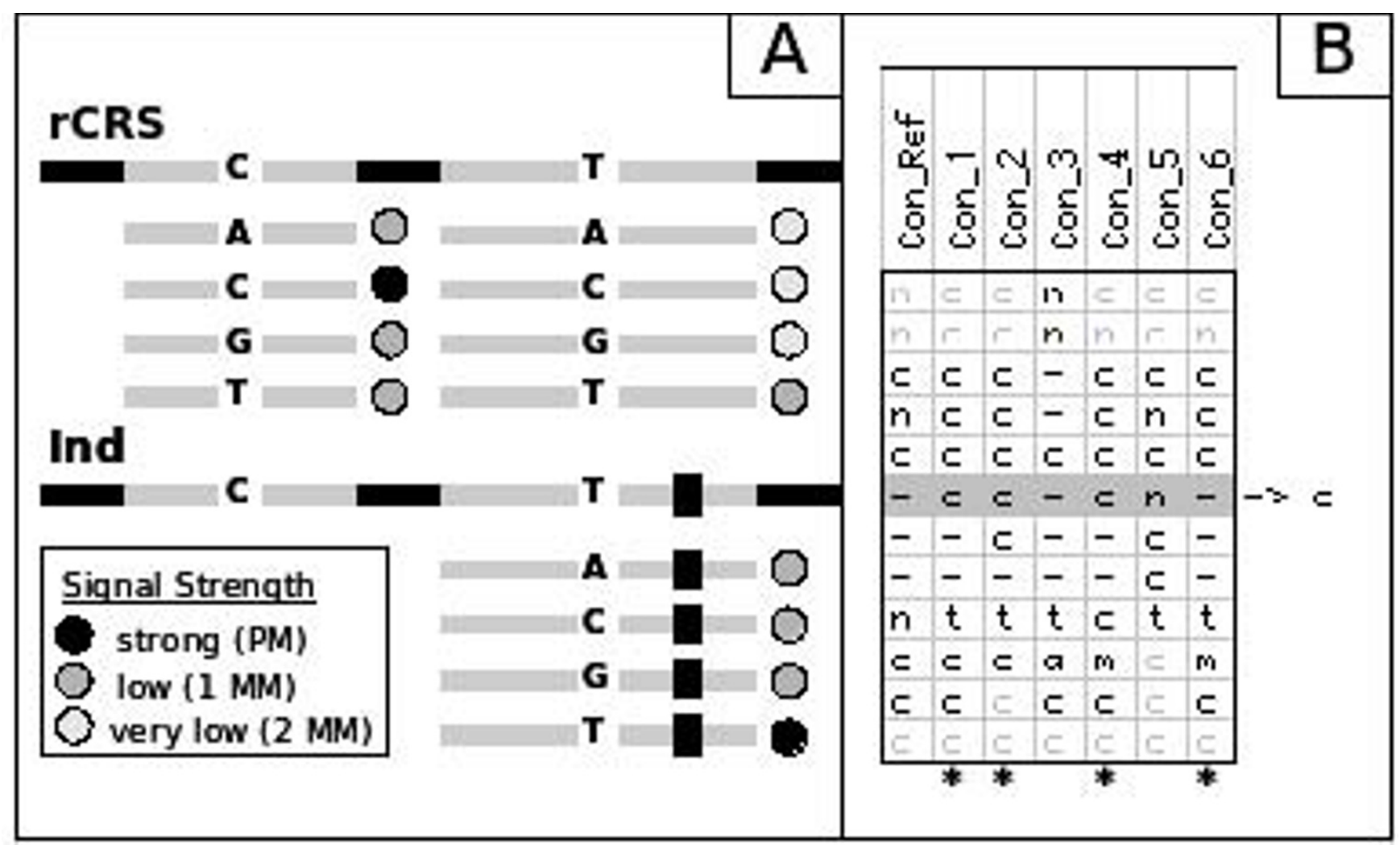

Figure I

Concept of multiple local context probes. (A) Three probe sets for 3 different sequence contexts are shown for one strand only. If an individual's (Ind) genome contains a nucleotide position different from the reference (rCRS) within I 2 nucleotide positions (nps) on either side of the interrogated position, only one of the alternative probes in the lower right corner will yield a strong hybridization signal. (B) Section of an alignment of additional fragments to reference sequence (left-most column). Due to the specified settings only fragments marked by a star fulfil the required reliability of no $\mathrm{N}$-calls within 3 nps on either side of the interrogated position.

imum likelihood. Forward and reverse strand information is combined by adding the respective (log-)likelihoods. A base call is issued if the difference between the two highest log likelihoods exceeds a given quality score threshold (QT). Otherwise the letter ' $\mathrm{N}$ ' is introduced into the sequence indicating that no conclusive evidence is available for this position.

ABACUS can be run in two modes to infer the genotype: The haploid mode only calls the four bases A, C, G, T or the letter N. It assumes the genotype to be homozygous, respectively in the case of mtDNA homoplasmic. The diploid mode calls all unsorted pairs of bases. Thus it detects both homozygous (homoplasmic) as well as heterozygous (heteroplasmic) genotypes. Note that ABACUS is designed for genotyping nuclear DNA, where heterozygous positions consist of two alleles present at a ratio of $1: 1$. For mtDNA the situation is different: As a cell may hold up to 100,000 mt genomes, allele proportions at a heteroplasmic site may vary over a wide range and even comprise more than two alleles, albeit rarely. Nevertheless, due to the lack of alternatives the ABACUS algorithm has been established as a quasi-standard for MitoChip base calling $[15,17]$ propose algorithms building on the output of ABACUS for the detection of heteroplasmy in mtDNA ignoring alternative context probes.

Here we focus on using the alternative context probes arrayed onto the MitoChip v2.0. We introduce ReseqChip, a novel, free and open source software implemented in Perl that exploits data from these alternative local mt genome context probes in the automated consolidation of base calls. ReseqChip does not use raw intensities as input but base calls for alternative local background probes. Ideally, intensities are strongest and likelihoods are highest for the correct base with a clear gap to the runner-up. This is typically observed, if the individual sequence matches the reference sequence both to the left and the right of the base in question in a window of 25bases in total (the size of the oligonucleotide probe). 


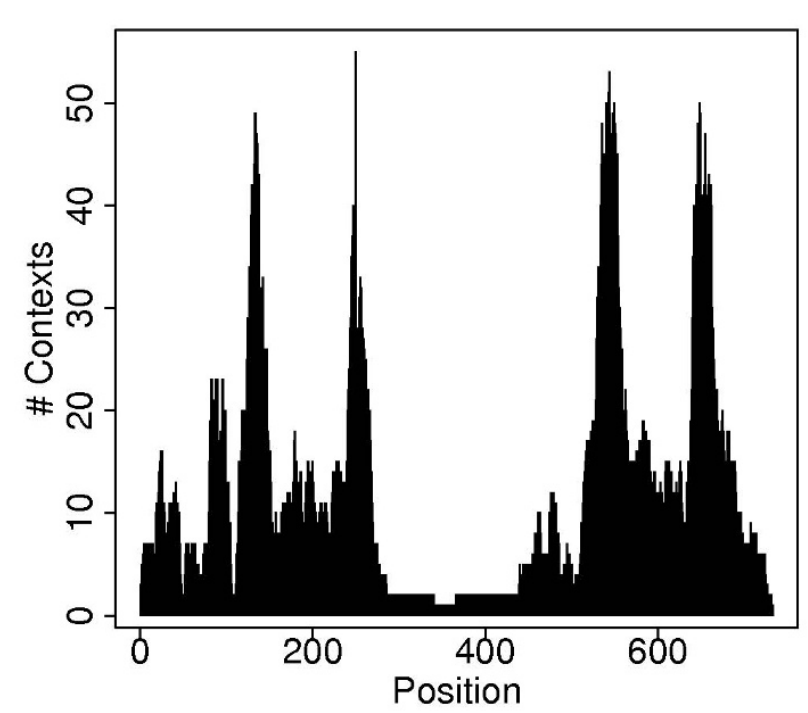

Figure 2

Different Contexts. Number of multiple contexts available by MitoChip v2.0 for the 733 nucleotide positions of the rCRS for which multiple local context probes are present. Note that the contexts of a certain position can vary at several of its 12 flanking bases up- and downstream of the center.

However, local accumulation of polymorphic sites around the base in question compromises hybridization signals.

Mismatches between the individual sequence and the probe sequence on the array are no longer restricted to the middle base but also occur in the flanking regions. Intensities do not only become weaker, they are also often more uniformly distributed across the four bases, resulting in many N-calls. ReseqChip consolidates these calls. It improves both sensitivity and accuracy compared to applying ABACUS alone.

\section{Implementation \\ Algorithm}

Input of ReseqChip are base calls from one of the two ABACUS implementations GSEQ or RATools (RA_Basecaller). The program accepts FASTA formated base caller output files for one or a series of chip experiments. Base calls need to be provided for the reference context as well as all additional contexts. In case of multiple local context probes for the same position the basecallers provide separate calls for each context. Our algorithm aims at identifying the best matching context.
We do this indirectly by excluding those probes that appear inadequate. Note, that if a local background is inadequate for a certain position, it will also be inadequate for neighboring positions. Hence, an indication of inadequacy is the accumulation of $\mathrm{N}$-calls. We investigate calls in neighborhoods of length $k$ around each sequence position and in each available context. Figure $1 \mathrm{~B}$ gives an example. In addition to calls obtained using the reference sequence context (left most column), base calls obtained from 6 alternative local context probes (Con_1-6) are available. Note that these alternatives can be related to sequence variants resulting not only from single-base substitutions, but also from insertions or deletions. For the latter, gaps need to be introduced to align homologous sequence positions. The row referring to the current sequence position in the re-sequencing run is highlighted in grey. Neighborhoods of size $k=3$ (not counting gaps) are shown as black letters. For probe filtering, the parameter $\max N$ is introduced, i.e. the maximum number of $\mathrm{N}$ calls allowed within the neighborhood. All sequence calls exceeding $\max N$ are then excluded from further analysis. In the example, $\max N$ was set at 0 , hence only probes producing no $\mathrm{N}$-calls were chosen. They are indicated by stars. In case the calls for the grey row are widely identical across the filtered columns, the actual call is based on majority voting. More precisely, we introduce the additional parameters $\min P$ and $\min U$. If more than $\min P$ probes remain after filtering and more than $\min U$ percent call the base $x$, where $x$ is the most frequently called base, then $x$ is included in the final sequence, otherwise the letter N. High values for minP ensure robust base calls, while high values for minU ensure a strong majority vote. In the example, $\min P$ was set to 2 , hence the 4 probes remaining after filtering are sufficient for base calling. Moreover, $\min U$ was set to $50 \%$ in the example. Since 3 out of 4 probes call the base, ReseqChip detects the insertion. ReseqChip is implemented in Perl and accepts a FASTA formated file that constitutes the base calls for one or a series of chip experiments as the output of the base caller. Base calls have to be provided for the genotypes with respect to the reference context as well as the additional contexts. The optimal parameter settings derived from the parameter training (see section Parameter Tuning) are provided in a separate parameter file (see Additional file 1) that can be used in conjunction with the test script (see section Availability and Requirements). It allows the user to choose a threshold for the maximum number of $\mathrm{N}$-calls per 100 nps or to define an own parameter set.

\section{Parameter Calibration}

ReseqChip operates on a number of parameters that need to be jointly calibrated. We use training data of known genotype (gold standard) and run ReseqChip on it. We judge parameter dependent performance based on: 
1. $d n$ - the number of discordant base calls between ReseqChip and the gold standard

2. $n c$ - the number of $\mathrm{N}$-calls

There is a trade-off between $d n$ and $n c$. Small $d c$ values lead to high $n c$ values and vice versa. We use a grid of values for one measure and minimize the second measure given the former. The user can then specify the maximal number of N-calls and ReseqChip runs with parameters minimizing the number of discordant call given the user specified restraint on the $\mathrm{N}$-calls. Vice versa, the user can set a restraint on $d c$ and receives parameters minimizing $n c$. We let the grid values of both $n c$ and $d c$ vary from 0 to 20 in steps of 0.1 .

\section{Results \\ MitoChip v2.0}

We have applied ReseqChip to data from the MitoChip (GeneChip ${ }^{\oplus}$ Mitochondrial Resequencing Array 2.0), a 36 $\mathrm{kb}$ oligonucleotide array. The array queries $16,544 \mathrm{nps}$ of the mt genome [10] in 25,413 $(25,032$ if one not distinguish between the order of PM and MM probes (see below)) different, unique contexts, with each context is represented by two probe quartets, one each for the forward and reverse strand, respectively. Each probe quartet consits of one probe that matches the sequence perfectly (PM) and three mismatched probes (MM) for the three alternative alleles. There are $11,529(11,910)$ redundant probes that serve as internal controls of hybridization reproducibility and provide 2 to 70 fold redundancy. That is, the array carries 36,942 probe quartets querying both rCRS [10] and common variants [23] thereof (Table 1). The numbers of interrogated variants, regardless of their contexts are summarized in Table 2 . These variants cover 233 different nps.

\section{DNA Samples and Chip Experiments}

$123 \mathrm{mt}$ genome sequences were analyzed using conventional dye-terminator sequencing as well as MitoChip v2.0 resequencing. Dye-terminator sequences constituted the gold standard that we aimed to recover using MitoChip v2.0 data. Among these sequences, 112 were sequenced completely and had been reported previously

Table I: MitoChip v2.0 (I)

\begin{tabular}{lccc}
\hline match & \# PQs & \# unique PQs (A) & \# unique PQs (B) \\
\hline rCRS & 18,824 & 16,544 & 16,544 \\
non rCRS & 18,118 & 8,869 & 8,488 \\
\hline Total & 36,942 & 25,413 & 25,032 \\
\hline
\end{tabular}

Number of probe quartets (PQs), unique PQs discerning $(A)$ and not discerning (B) the probes representing the $\mathrm{PM}$ and the three MMs that match the rCRS and variations of them, respectively.
Table 2: MitoChip v2.0 (II)

\begin{tabular}{lcc}
\hline Type of Variation & \# unique & \# all \\
\hline Single-base substitution & 246 & 1365 \\
Deletion & 28 & 102 \\
Insertion & 20 & 94 \\
\hline Total & 294 & 1561 \\
\hline
\end{tabular}

Number of variations that are interrogated by the MitoChip v2.0 additional probes.

$[19,24]$, while 19 were analyzed only with respect to the HVS I and II. In addition, pseudo-heteroplasmatic samples were generated for $11 \mathrm{mt}$ genomes by mixing DNA at a ratio of 1:1. All pairwise combinations of mixed DNA where generated in two disjoint groups of 5 and 6 individuals resulting in 10 and 15 controlled heteroplasmic samples, respectively. For these individuals gold standard sequences by dye-terminator sequencing using a published protocol [25] were available. Combining this data with the mixing protocols yielded gold standard genotypes for the artificial heteroplasmic sites. In total we analyzed 148 genomes, whereof 25 were artificial mixtures. This data harbored 172 variants at 159 nps, from which 13 were triallelic. Five of the variants were insertions, 3 were deletions. The total numbers of variable sites were 1190, 246 and 15 for substitutions, insertions and deletions, respectively. The artificially created mixtures contained 205 single nucleotide substitution and 14 insertion type heteroplasmies at 41 variable sites. The polymorphic sites as discovered by dye-terminator sequencing are summarized in Table 3. For every genome we generated base calls with GSEQ for Quality Threshold (QT) values of $3,6,9$ and 12 using both haploid and diploid settings.

Relevant for our analysis were only those nps, that were interrogated by at least one alternative local context probe set. There were a total of $733 \mathrm{nps}$ that met this criterion. Twenty additional nps cover insertions. In total 21,121 probe quartets interrogated these 753 positions, whereof 9,602 were unique. On average about 13 different contexts were assigned to each of the 753 position. Since the 125 contiguous nps around the center of Figure 2 were covered by only one additional context and probes only for less than $1.1 \%$ of the contexts, we excluded these positions and restricted our further analysis to the remaining

Table 3: Dataset (I)

\begin{tabular}{lccc}
\hline & \# Inds & \# Pos/Ind & \# Pos \\
\hline Total & 148 & & $89,244(92,204)$ \\
Train & 49 & $603(623)$ & $29,547(30,527)$ \\
Test & 99 & & $59,697(61,677)$
\end{tabular}

Number of nps involved in the training and test data w/o and with (in brackets) insertions. 
608 (628) nps. We further excluded nps 107-111 of the rCRS owing to persistent wrong hybridization signal, resulting from a repetitive sequence pattern, which reduced the total number of positions to 603 (623). We randomly selected 49 samples to form a training set for calibrating parameters and collected the remaining 99 for evaluating genotyping performance. The total number of positions involved in the training and testing process and the number of variants and variable sites are listed in Tables 3 and 4 .

\section{Parameter Calibration}

We ran ReseqChip on the training data for various parameter constellations. Discrepancies between MitoChip and the gold standard were considered array-based sequencing errors and nps, for which no call had been generated were counted as $\mathrm{N}$-calls. Let $d c$ be the number of sequencing errors and $n c$ the number of N-calls. The goal was to keep both measures small. ReseqChip builds on the base calls generated by GSEQ, which depend on the Quality Threshold QT. Hence QT is an additional parameter that needs to be jointly calibrated with the ReseqChip internal parameters. We repeated our analysis using a range of discrete $Q T$ values $(Q T=3,6,9,12)$. Moreover, we varied the four ReseqChip intrinsic parameters, namely the neighborhood size $(k=0,1, \ldots, 12)$, the maximal number of $\mathrm{N}$ calls for probe filtering $(\max N=0,1, \ldots, 5)$, the minimal number of high quality probes $(\min P=1, \ldots, 11)$, and the uniqueness threshold $(\min U=30 \%, \ldots, 100 \%)$. In total, 27,456 parameter combinations were tested. The challenge was to jointly tune this set of parameters so that acceptable performances were obtained for both, the number of $\mathrm{N}$-calls and sequencing errors.

Figure 3 shows the $n c$ and $d c$ performance for all 27,456 constellations of parameters assuming a haploid and a diploid model, respectively. Note that there is a trade-off

Table 4: Dataset (II)

\begin{tabular}{lccccc}
\hline & & Sub & Del & Ins & Total \\
\hline Homoplasmic & All & $985(166)$ & $15(3)$ & $246(3)$ & $1246(172)$ \\
& Train & $336(110)$ & $4(3)$ & $86(2)$ & $426(112)$ \\
& Test & $649(143)$ & $11(2)$ & $160(3)$ & $820(148)$ \\
\hline \multirow{2}{*}{ Heteroplasmic } & All & $205(39)$ & 0 & $14(2)$ & $219(4 I)$ \\
& Train & $75(36)$ & 0 & $6(2)$ & $81(38)$ \\
& Test & $130(39)$ & 0 & $8(2)$ & $138(41)$ \\
\hline \multirow{2}{*}{ Total } & All & $1190(166)$ & $15(3)$ & $260(3)$ & $1465(172)$ \\
& Train & $411(116)$ & $4(3)$ & $92(3)$ & $507(122)$ \\
& Test & $779(143)$ & $11(2)$ & $168(3)$ & $958(148)$ \\
\hline
\end{tabular}

Number of variable sites in the dataset are listed and stratified by type of variation and homoplas-mic/heteroplasmic state. (In brackets the number of distinct SNPs are given.) between $\mathrm{N}$-calls and sequencing errors. They cannot be optimized simultaneously. For example, increasing maxN lead to a smaller number of $\mathrm{N}$-calls, albeit at the expense of more sequencing errors, while lowering $\min U, \min P$ and $k$ has the opposite effect. Depending on the application, the balance between $n c$ and $d c$ can be adjusted. ReseqChip allows the user to specify a bound on either $n c$ or $d c$ and then uses the training data to optimize the parameters with respect to the second error measure. For example, if bounds are set to $n c$ we use the data in Figure 3 for calculating the minimum $d c$ for increasing $n c$. Optimal settings are indicated by the red dots at the bottom boundary of the cloud. For these points, the x-axis represents the bound to $n c$, while the y-axis gives the optimal $d c$ performance achievable under this constraint. The optimal parameter constellations as obtained by the training procedure are listed in Additional file 1.

\section{Parameter Testing}

Note that the performances indicated by the red dots in Figure 3 are training set performances. They might be overoptimistic estimates of performance for independent data sets. We evaluated the performance of ReseqChip on independent data using the optimal parameter settings derived from the training data. Figure 4 shows for substitutions/deletions only the results that were obtained applying the various parameter constellations to the test sets for the haploid and the diploid model, respectively. The circles and triangles display the average numbers of discordant (dc) and noncalled (nc) bases normalized to 100 nps that were observed upon inclusion (using ReseqChip) and exclusion, respectively, of the local context probes applying primary base calls only generated by GSEQ for QT values of 3, 6, 9, 12, 18 and 24. Evidently, ReseqChip reduces considerably both the number of sequencing errors and the number of $\mathrm{N}$-calls in comparison to a sequence assembly ignoring the alternative local context probes.

\section{Discussion}

ReseqChip allows for the automated consolidation of multiple base calls from alternative local $\mathrm{mt}$ genome context probes into a single call, thereby improving the accuracy of mt genome resequencing, while simultaneously reducing the number of $\mathrm{N}$-calls. Based on primary base calls only, it shall be feasible to use ReseqChip with any base calling algorithm and re-sequencing array carrying multiple local context probes. However, this remains to be demonstrated.

Exemplified by the MitoChip v2.0 and the GSEQ basecaller, we demonstrated that an additional sequence consolidation step for analyzing the additional base calls using ReseqChip improved both the accuracy and the call rate. Interestingly, almost all optimal parameter constella- 
Hap

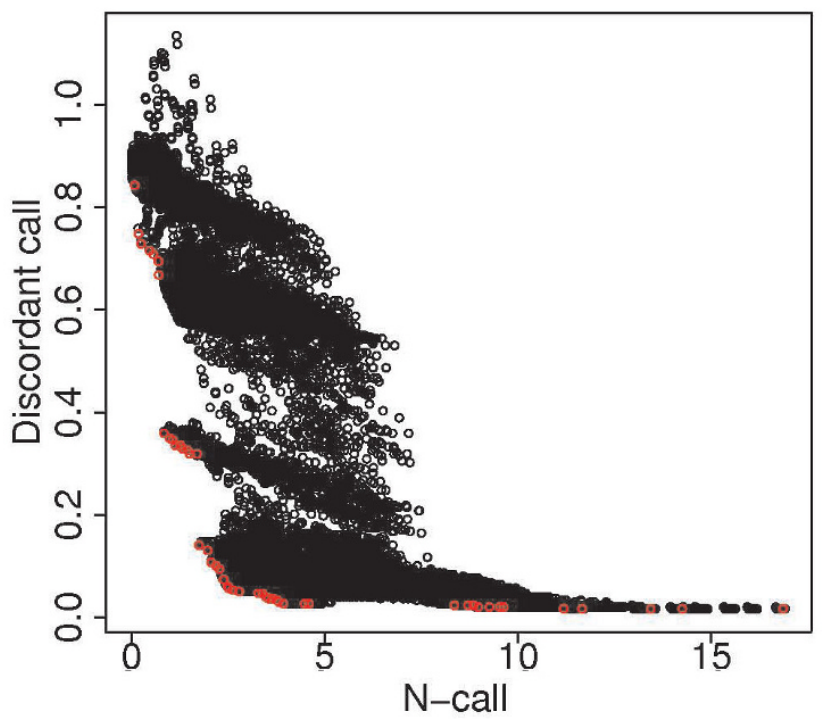

Hap

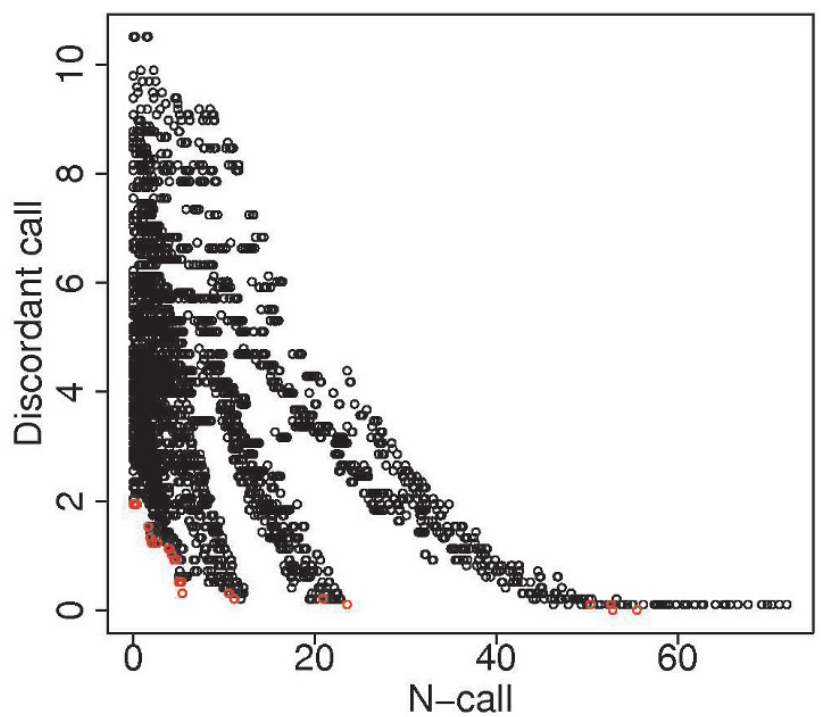

Dip

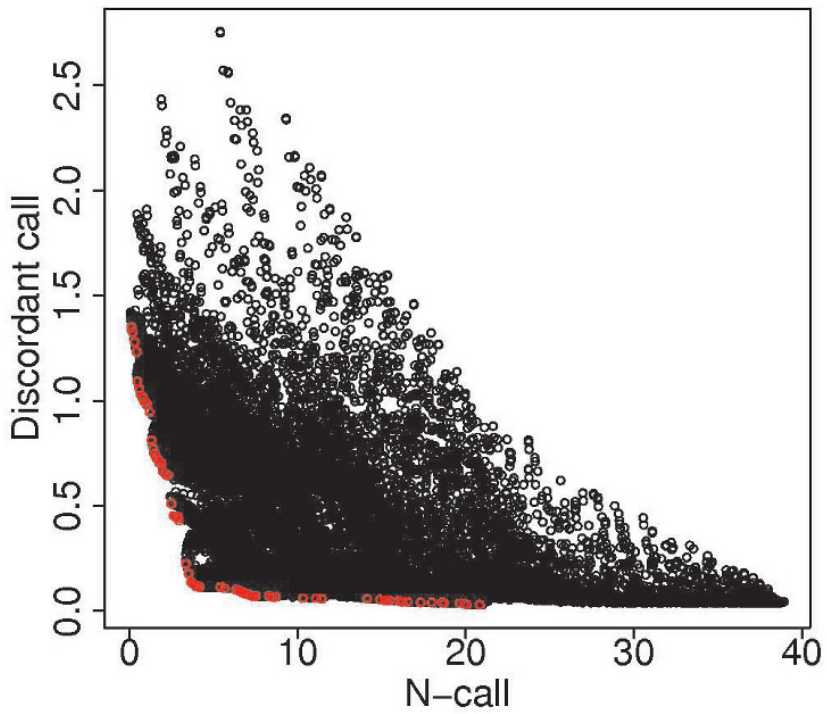

Dip

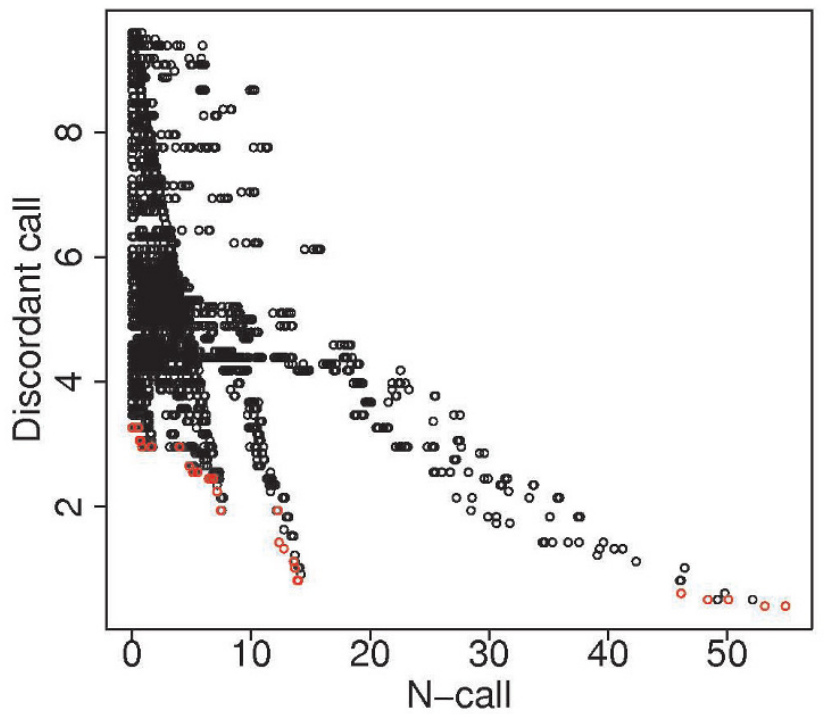

Figure 3

Performance on Train Data. The plots show the performance of the 27,456 different parameter constellations in terms of number of sequencing errors and $\mathrm{N}$-calls for substitutions/deletions (top) and insertions (bottom) for haploid (left) and diploid (right) settings of GSEQ.

tions incorporate a $Q T$ value of 3 . This indicates that the algorithm performs best with a low number of N-calls, which in turn gives a reasonable number of possibly conflicting local contexts that can be evaluated. Vice versa, a sequence assembly based on less but more accurate base calls is inferior.
Despite the improvements in call rate and accuracy achieved by means of ReseqChip, it seems impossible to achieve call rates similar to those of conventional sequencing at equal accuracy for at least two reasons: First, array-based resequencing is facing inherent difficulties with respect to variability of the target sequence. There are 

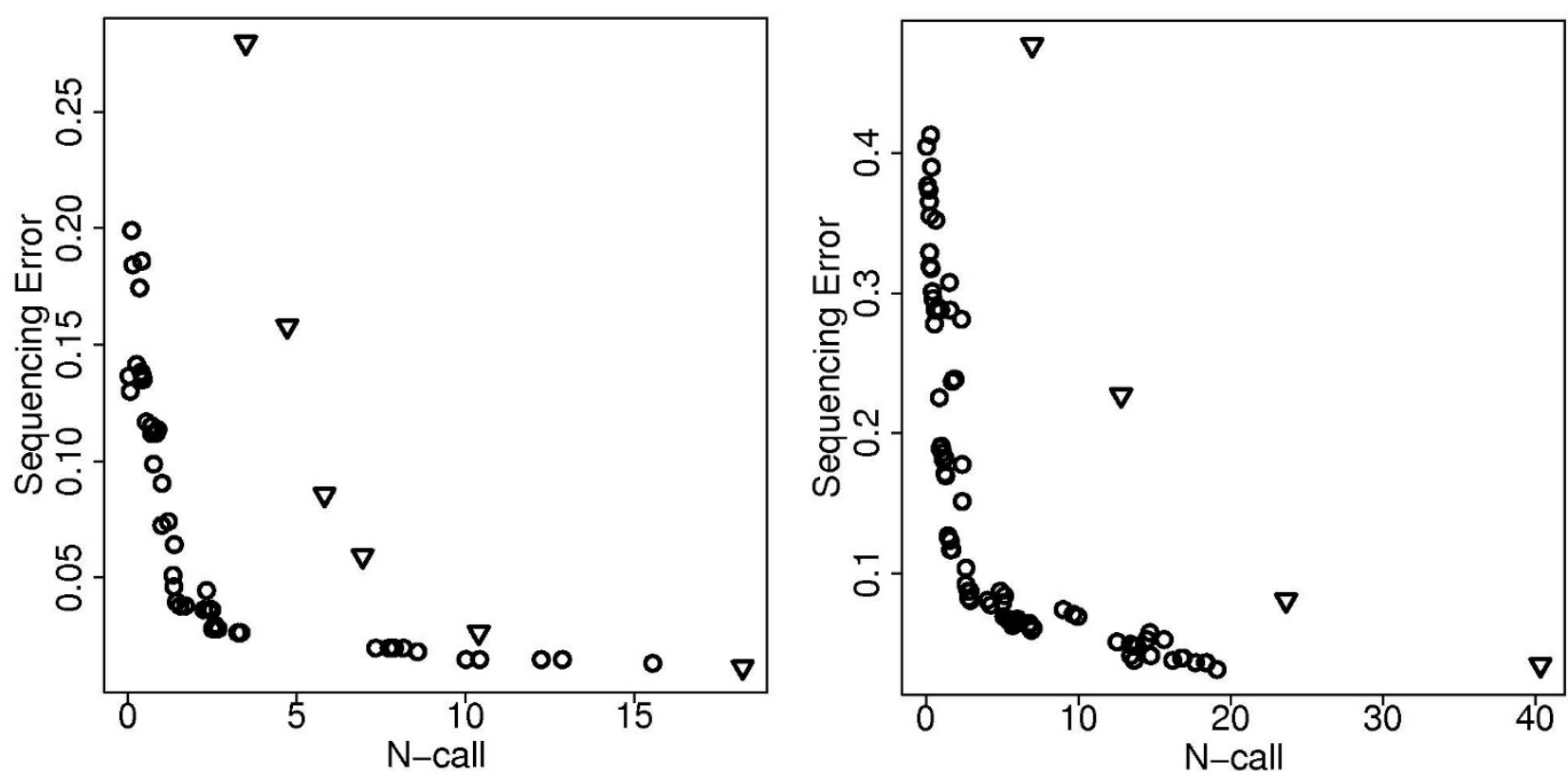

\section{Figure 4}

Performance on Test Data. The performance of ReseqChip for the test dataset based on the number of sequencing errors and $\mathrm{N}$-calls normalized to 100 nps is plotted for substitutions/deletions with haploid (left) and diploid (right) settings of GSEQ. The circles represent the performance of the optimal parameter constellations obtained from the training. The triangles represent the performance of running GSEQ for QT values of 3,6,9,12,18,24 (but without subsequent sequence assembly by ReseqChip). In the diploid settings only QT values of 3,6,9, I2 are shown.

almost always individual target sequences that do not match some contexts well. Secondly, the principles underlying the hybridization of target sequences to surfaceattached probes are still not completely understood. Though discrimination between signal intensities of PM and MM in DNA/DNA hybridization is relatively precise, some PM and MM probes show an unfavorable relation between their signal intensities. This is in particular due to base composition of probe sequence, neighboring bases of PM and MM, and the MM itself [26]. This implies, however, if several probe quartets probing the same or a slightly different local context yield primary base calls that are persistently wrong, then also the consensus call calculated by ReseqChip is wrong. That contributes to the fact that the number of $\mathrm{N}$ - and discordant calls cannot become zero.

Our results also show that base calls generated in a diploid model give less accurate calls and more N-calls. For this reason, we have also not pursued allele ratios other than $1: 1$, as it is obvious that accuracy will decrease with decreasing frequency of the variant allele. We believe that this limitation can be approached only at the level of the basecaller.

\section{Conclusions}

We provide ReseqChip, a freely available software for the automated generation of consensus base calls from resequencing arrays that carry multiple local context probes on the MitoChip v2.0. ReseqChip processes both homozygous (homoplasmic) as well as heterozygous (heteroplasmic) base calls of many chips, hence enabling high-throughput generation of sequence data. ReseqChip significantly improves both accuracy and call rate.

\section{Availability and Requirements}

Project name: ReseqChip

Project home page: The project is part of the live branch of the BioPerl project (bioperl-live): http://code.openbio.org/svnweb/index.cgi/bioperl/browse/bioperl-live/ trunk/Bio/Microarray/Tools/

Availability: the BioPerl live distribution can be downloaded via anonymous svn checkout: svn co svn:// code.open-bio.org/bioperl/bioperl-live/trunk bioperl-live

The module path for ReseqChip is Bio/Microarray/Tools. A test script (t/Microarray/Tools/ReseqChip.t) together 
with test data and optimized parameter set are provided in separate files. The latter files reside in $\mathrm{t} / \mathrm{data} / \mathrm{and}$ carry the prefix "ReseqChip ".

Operating systems: Cross platform

Programming language: Perl

Other requirements: Perl interpreter, BioPerl 1.4 or higher, Statistics-Frequency-0.03 from CPAN http:// search.cpan.org/dist/Statistics-Frequency/

License: GNU GPL

\section{Authors' contributions}

Critical revision of the manuscript for important intellectual input: RS and PJO. Technical and material support: HN and WP. Study supervision: PJO. Study concept and design: PJO and RS. Software development: MT. Analysis of data and data interpretation: MT, CL, RS, PJO. Drafting of the manuscript: MT. All authors read and approved the final manuscript.

\section{Additional material}

\section{Additional file 1}

Optimal parameter settings. A CSV file listing the optimal parameter settings obtained from the training for haploid/diploid model and Single base substitutions-Deletions/Insertions variants.

Click here for file

[http://www.biomedcentral.com/content/supplementary/14712105-10-440-S1.CSV]

\section{Acknowledgements}

MT, CL, RS and PJO are supported by the Bavarian Genome Research Network (BayGene). In addition, MT and PJO acknowledge support from the National Institutes of Health (CAI00352). WP recieved support from the Austrian FWF Translational Research programme (TR397). We thank Barbara Herte for dye-terminator sequencing and long-range PCR amplification of $\mathrm{mt}$ DNA. Gabriela Huber, Bettina Zimmermann and Liane Fendt (all GMI Innsbruck) are acknowledged for full mtDNA sequence analysis. MitoChip v2.0 array analysis was performed by the Center of Excellence for Fluorescent Bioanalytics (KFB) at the University of Regensburg. We thank Michael Wulfert (Department of Haematology, Oncology and Clinical Immunology at the University of Duesseldorf) for providing DNA material.

\section{References}

I. Henn BM, Gignoux CR, Feldman MW, Mountain JL: Characterizing the Time Dependency of Human Mitochondrial DNA Mutation Rate Estimates. Mol Biol Evol 2009, 26:2 17-230.

2. Yakes FM, Van Houten B: Mitochondrial DNA damage is more extensive and persists longer than nuclear DNA damage in human cells following oxidative stress. Proc Natl Acad Sci USA 1997, 94:514-519.

3. Chinnery PF, Schon EA: Mitochondria. J Neurol Neurosurg Psychiatry 2003, 74(9): I I88-II99.

4. A Chatterjee EM, Sidransky D: Mitochondrial DNA mutations in human cancer. Oncogene 2006, 25:4663-4674.
5. Wallace DC: Diseases of the Mitochondrial DNA. Annual Review of Biochemistry 1992, 61: I 175- I2 12. [PMID: 1497308]

6. Budowle B, Allard MW, Wilson MR, Chakraborty R: FORENSICS AND MITOCHONDRIAL DNA: Applications, Debates, and Foundations. Annual Review of Genomics and Human Genetics 2003, 4:II9-14I.

7. Pennisi E: PALeogenetics: The Dawn of Stone Age Genomics. Science 2006, 3 I 4(5802): 1068-107|.

8. Kivisild T, Shen P, Wall DP, Do B, Sung R, Davis K, Passarino G, Underhill PA, Scharfe C, Torroni A, Scozzari R, Modiano D, Coppa A, de Knijff P, Feldman M, Cavalli-Sforza LL, Oefner PJ: The Role of Selection in the Evolution of Human Mitochondrial Genomes. Genetics 2006, 172:373-387.

9. Maitra A, Cohen Y, Gillespie SE, Mambo E, Fukushima N, Hoque MO, Shah N, Goggins M, Califano J, Sidransky D, Chakravarti A: The Human MitoChip: A High-Throughput Sequencing Microarray for Mitochondrial Mutation Detection. Genome Res 2004, 14(5):8I2-8I9.

10. Andrews R, Kubacka I, PF C, RN L, Turnbull D, Howell N: Reanalysis and revision of the Cambridge reference sequence for human mitochondrial DNA. Nat Genet 1999, 23:147.

II. Zhou S, Kassauei K, Cutler DJ, Kennedy GC, Sidransky D, Maitra A, Califano J: An Oligonucleotide Microarray for High-Throughput Sequencing of the Mitochondrial Genome. J Mol Diagn 2006, 8(4):476-482.

12. Sui G, Zhou S, Wang J, Canto M, Lee EE, Eshleman JR, Montgomery EA, Sidransky D, Califano JA, Maitra A: Mitochondrial DNA mutations in preneoplastic lesions of the gastrointestinal tract: $A$ biomarker for the early detection of cancer. Mol Cancer 2006, $5: 73+$.

13. Zhou S, Kachhap S, Sun W, Wu G, Chuang A, Poeta L, Grumbine L, Mithani SK, Chatterjee A, Koch W, Westra WH, Maitra A, Glazer C, Carducci M, Sidransky D, McFate T, Verma A, Califano JA: Frequency and phenotypic implications of mitochondrial DNA mutations in human squamous cell cancers of the head and neck. Proceedings of the National Academy of Sciences 2007, 104( 18):7540-7545.

14. Kassauei K, Habbe N, Mullendore ME, Karikari CA, Maitra A, Feldmann G: Mitochondrial DNA Mutations in Pancreatic Cancer. Int J Gastrointest Cancer 2006, 37(2-3):57-64.

15. Mithani SK, Smith IM, Zhou S, Gray A, Koch WM, Maitra A, Califano J: Mitochondrial Resequencing Arrays Detect Tumor-Specific Mutations in Salivary Rinses of Patients with Head and Neck Cancer. Clin Cancer Res 2007, 13(24):7335-7340.

16. Mithani S, Smith I, Topalian S, JA C: Nonsynonymous somatic mitochondrial mutations occur in the majority of cutaneous melanomas. Melanoma Res 2008, I8(3):214-9.

17. Jakupciak J, Maggrah A, Maragh S, Maki J, Reguly B, Maki K, Wittock R, Robinson K, Wagner P, Thayer R, Gehman K, Gehman T, Srivastava S, Ngom A, Dakubo G, Parr R: Facile whole mitochondrial genome resequencing from nipple aspirate fluid using MitoChip v2.0. BMC Cancer 2008, 8:95.

18. Vallone P, Jakupciak J, Coble M: Forensic application of the affymetrix human mitochondrial resequencing arrays. Forensic Science International: Genetics 2007, I: 196-198.

19. Hartmann A, Thieme M, Nanduri LK, Stempfl T, Moehle C, Kivisild T, Oefner PJ: Validation of microarray-based resequencing of 93 worldwide mitochondrial genomes. Hum Mutat 2009, 30:115-122.

20. Lévêque $M$, Marlin S, Jonard L, Procaccio V, Reynier P, Amati-Bonneau $P$, Baulande $S$, Pierron D, Lacombe D, Duriez F, Francannet $C$, Mom T, Journel H, Catros H, Drouin-Garraud V, Obstoy MF, Dollfus H, Eliot MM, Faivre L, Duvillard C, Couderc R, Garabedian EN, Petit $C$, Feldmann D, Denoyelle F: Whole mitochondrial genome screening in maternally inherited non-syndromic hearing impairment using a microarray resequencing mitochondrial DNA chip. Eur J Hum Genet 2007, I 5: I | 45- I I55.

21. Maragh S, Jakupciak J, Wagner P, Rom W, Sidransky D, Srivastava S, O'Connell C: Multiple strand displacement amplification of mitochondrial DNA from clinical samples. BMC Medical Genetics 2008, 9:7.

22. Cutler DJ, Zwick ME, Carrasquillo MM, Yohn CT, Tobin KP, Kashuk C, Mathews DJ, Shah NA, Eichler EE, Warrington JA, Chakravarti A: High-Throughput Variation Detection and Genotyping Using Microarrays. Genome Res 2001, I I:1913-1925. 
23. Monson KL, Miller KWP, DiZinno JA, Budowle B: The mtDNA population database: an integrated software and database resource. Forensic Science Communications 2002, 4(2):.

24. Wulfert M, Küpper AC, Tapprich C, Bottomley SS, Bowen D, Germing $U$, Haas R, Gattermann N: Analysis of mitochondrial DNA in 104 patients with myelodysplastic syndromes. Exp Hematol 2008, 36(5):577-586.

25. Fendt L, Zimmermann B, Daniaux M, Parson W: Sequencing strategy for the whole mitochondrial genome resulting in high quality sequences. BMC Genomics 2009, 10:139.

26. Naiser T, Ehler O, Kayser J, Mai T, Michel W, Ott A: Impact of point-mutations on the hybridization affinity of surfacebound DNA/DNA and RNA/DNA oligonucleotide-duplexes: Comparison of single base mismatches and base bulges. $B M C$ Biotechnology 2008, 8:48.

Publish with Bio Med Central and every scientist can read your work free of charge

"BioMed Central will be the most significant development for disseminating the results of biomedical research in our lifetime. "

Sir Paul Nurse, Cancer Research UK

Your research papers will be:

- available free of charge to the entire biomedical community

- peer reviewed and published immediately upon acceptance

- cited in PubMed and archived on PubMed Central

- yours - you keep the copyright

Submit your manuscript here:

http://www.biomedcentral.com/info/publishing_adv.asp
BioMedcentral 